03

\title{
О расщеплении субциклового импульса при когерентном распространении в резонансной среде
}

\author{
(C) P.М. Архипов ${ }^{1,2}$, Н.Н. Розанов ${ }^{2}$ \\ ${ }^{1}$ Санкт-Петербургский государственный университет, \\ 199034 Санкт-Петербург, Россия \\ ${ }^{2}$ Университет ИТМО, \\ 197101 Санкт-Петербург, Россия \\ e-mail: arkhipovrostislav@gmail.com
}

Поступила в редакцию 13.01.2018 г.

В работе теоретически изучается динамика распространения униполярного, субциклового импульса большой элекрической площади (интеграл от величины электрического поля по времени) при когерентном распространении в нелинейной двухуровневой резонансной среде. Показана возможность расщепления импульса на отдельные составляющие, каждый из которых ведет себя подобно импульсу самоиндуцированной прозрачности. Данное явление аналогично хорошо известному явлению расщепления $4 \pi$ - и $6 \pi$-импульса на пары $2 \pi$-импульсов самоиндуцированной прозрачности, которое имеет место в случае распространения длинных импульсов, когда применимо понятие площади импульса, и выполняется теорема площадей.

DOI: 10.21883/OS.2018.05.45954.9-18

\section{Введение}

В настоящее время стали доступны предельнокороткие импульсы (ПКИ) с длительностью порядка периода колебаний световой волны в различных спектральных диапазонах, см. обзоры [1-3]. Изучение взаимодействия таких импульсов с веществом открыло уникальные возможности в нелинейной оптике. Так, стало возможно управлять динамикой волновых пакетов в веществе и наноструктурах [4-7], ускорять заряженные частицы [8], изучать динамику солитонов в нелинейных средах и генерировать излучение в широком спектральном интервале (генерация суперконтинуума) [9-12] и т.д.

Когерентное взаимодействие ПКИ с резонансной средой возникает,когда длительность ПКИ намного меньше времен релаксации поляризации и разности заселенностей $T_{1}$ в резонансной среде [13-16], и при этом возможно явление самоиндуцированной прозрачности (СИП). Такой $2 \pi$-импульс СИП распространяется в резонансной среде без потерь. Использование СИП открывает новые возможности в генерации ПКИ импульсов аттосекундной длительности в резонансных средах [17-28], возможность наведения в среде решеток инверсии и сверхбыстрого управления ими с помощью последовательности ПКИ, не перекрывающихся в среде [29-33].

Основным понятием, широко применяемым при описании когерентных резонансных взаимодействий коротких импульсов с веществом, является понятие площади импульса

$$
\Theta(z) \equiv \frac{d_{12}}{\hbar} \int_{-\infty}^{\infty} \varepsilon(z, t) d t
$$

$\left(d_{12}\right.$ - дипольный момент перехода, $\varepsilon(t)-$ медленная огибающая импульса) [13-16]. Эволюция площади импульса при когерентном распространении длинных импульсов в резонансных средах подчиняется теореме площадей Мак-Колла и Хана [13-16]. Однако, как показывают исследования, когда длительность импульса сравнима с периодом колебания световой волны, возможны отклонения от теоремы площадей, и понятие площади импульса (1) становится не применимым [17-20,29-39].

В особенности понятие площади импульса не применимо в случае так называемых униполярных импульсов, электрическое поле в которых не меняет знак в течение длительности импульса. В таких импульсах отсутствует несущая частота, и понятие огибающей теряет смысл. Возможность получения таких импульсов привлекает внимание в связи с однонаправленным воздействием на заряженные частицы, что может быть использовано в управлении динамикой волновых пакетов в веществе, см. [17-28] и обзоры $[40,41]$. В случае униполярных импульсов говорят об электрической площади импульса - интеграле от напряженности электрического поля по времени, сохраняющем, в рамках одномерного рассмотрения, величину при любых продольных координатах [42]

$$
S_{E} \equiv \int_{-\infty}^{\infty} E(t) d t
$$

В работе [43] было показано существование солитонного решения уравнений Максвелла-Блоха в виде униполярного субциклового импульса, форма которого представляет собой гиперболический секанс. Если электрическая площадь такого импульса (2) равна $2 \pi$, т. е.действие такого импульса на среду аналогично действию импульса СИП. Столкновительная динамика и возникновение субцикловых солитонов при когерентном 
распространении длинных биполярных импульсов в резонансной среде в режиме СИП проанализированы в работах $[17,21-28,44]$. Однако когерентное распространение униполярных субцикловых импульсов на сегодняшний день слабо изучено, см., например, [21,31,32,44,45].

Хорошо известно, что при когерентном распространении длинных импульсов большой площади, определяемой (1) и кратной четным значениям $n \pi(n=4,6, \ldots)$, в резонансной среде возможно расщепление такого импульса на пары $2 \pi$-импульсов самоиндуцированной прозрачности [13-16]. Например, импульс входной площади, определяемой формулой (1) и равной $4 \pi$, расщепляется на пару $2 \pi$-импульсов СИП, распространяющихся с разной скоростью [13-16]. Это явление имеет место также в случае распространения одноциклового биполярного импульса в резонансной среде [35]. Однако в случае униполярного субциклового импульса, как упоминалось выше, понятие площади импульса (1) неприменимо, и теорема площадей нарушается [35]. В этом случае необходимо говорить об электрической площади импульса (2). Поэтому естественно встает вопрос об изучении динамики когерентного распространения униполярных субцикловых импульсов в среде.

В связи с этим в настоящей работе изучается распространение униполярного субциклового импульса большой электрической площади в нелинейной резонансной среде. Показано, что, несмотря на неприменимость теоремы площадей, для таких импульсов также возникает расщепления импульса с входной электрической площадью $4 \pi$ на пару импульсов. Каждый из них действует на среду аналогично действию $2 \pi$-импульсов СИП, меняя инверсию среды и возвращая среду в основное состояние после окончания действия импульсов. Эта ситуация справедлива, когда длительность входного импульса $\tau_{p}$ меньше периода $T_{0}=\frac{2 \pi}{\omega_{0}}$ собственных колебаний резонансного перехода среды с частотой $\omega_{0}$, т. е.выполнено условие $\omega_{0} \tau_{p} \ll 1$.

\section{Результаты численных экспериментов}

Для изучения распространения субциклового импульса в резонансной среде применялась система уравнений Максвелла-Блоха. Ввиду малой длительности возбуждающих импульсов в ней не используются приближение медленно меняющихся амплитуд и приближение вращающейся волны. Данная система уравнений имеет вид

$$
\begin{gathered}
\frac{\partial \rho_{12}(z, t)}{\partial t}=-\frac{\rho_{12}(z, t)}{T_{2}}+i \omega_{0} \rho_{12}(z, t)-\frac{i}{\hbar} d_{12} E(z, t) n(z, t) \\
\frac{\partial n(z, t)}{\partial t}=-\frac{n(z, t)-n_{0}}{T_{1}}+\frac{4}{\hbar} d_{12} E(z, t) \operatorname{Im} \rho_{12}(z, t) \\
P(z, t)=2 N_{0} d_{12} \operatorname{Re}\left(\rho_{12}\right) \\
\frac{\partial^{2} E(z, t)}{\partial z^{2}}-\frac{1}{c^{2}} \frac{\partial^{2} E(z, t)}{\partial t^{2}}=\frac{4 \pi}{c^{2}} \frac{\partial^{2} P(z, t)}{\partial t^{2}}
\end{gathered}
$$

Система (3)-(6) содержит следующие величины: $P$ поляризация среды, $N_{0}$ - концентрация активных центров, $E$ - напряженность электрического поля с фиксированной линейной поляризацией, $c-$ скорость света в вакууме, $\hbar-$ приведенная постоянная Планка, $\omega_{0}-$ частота резонансного перехода среды $\left(\lambda_{0}=2 \pi c / \omega_{0}-\right.$ длина волны резонансного перехода), $n_{0}$ - разность заселенностей двух рабочих уровней при отсутствии электрического поля $\left(n_{0}=1\right.$ для поглощающей среды). Уравнения (3), (4) описывают эволюцию недиагонального элемента матрицы плотности $\rho_{12}$ и разности $n \equiv \rho_{11}-\rho_{22}$ между диагональными элементами матрицы плотности, которая имеет смысл разности заселенности (инверсии) между основным и возбужденным состояниями двухуровневой системы. Поляризация среды связана с недиагональным элементом матрицы плотности $\rho_{12}$ по формуле (5). Среда описывается уравнениями (3)-(5) в двухуровневом приближении с использованием формализма матрицы плотности.

Система уравнений Максвелла-Блоха (3)-(6) активно используется в литературе для изучения когерентного распространения ПКИ в резонансной среде [17-46] вплоть до субцикловой длительности. В случае импульса субцикловой длительности применение двухуровневого приближения может нарушаться. Однако, как показывают расчеты, основные особенности когерентного распространения ПКИ в двухуровневых средах сохраняются и в многоуровневых средах [22-26, 33] и с учетом неоднородного уширения [47]. Сохраняются, в частности, основные свойства генерации ПКИ за счет СИП. Поэтому в настоящей работе будет для простоты использовано также двухуровневое приближение, а неоднородным уширением спектрального перехода мы будем пренебрегать. Мы пренебрегаем также дифракцией излучения, что оправдано для длин распространения пучков, меньших дифракционной длины.

Как и в работах [29-33], уравнения для матрицы плотности (3), (4) решались методом Рунге-Кутта 4-го порядка. Волновое уравнение (6) решалось методом конечных разностей. Пространственная область интегрирования имела длину $L=30 \lambda_{0}$. Резонансная среда располагалась вдоль оси $z$ в центре области между точками $z_{1}=2 \lambda_{0}$ и $z_{2}=25 \lambda_{0}$. Слева направо в среду посылался униполярный субцикловый импульс в форме гиперболического секанса:

$$
E(t)=E_{0} \operatorname{sech}\left[\frac{t}{\tau_{p}}\right] .
$$

Была проведена серия численных экспериментов при различных параметрах среды, длительностях и амплитудах входного импульса. Проведенные расчеты показали, что импульс с входной электрической площадью $4 \pi$ расщепляется по мере распространения на пару импульсов, каждый из которых ведет себя подобно $2 \pi$-импульсу, меняя инверсию среды и возвращая среду обратно в основное состояние.

Пример эволюции импульса с входной электрической площадью $4 \pi$ по мере распространения в двухуровне- 


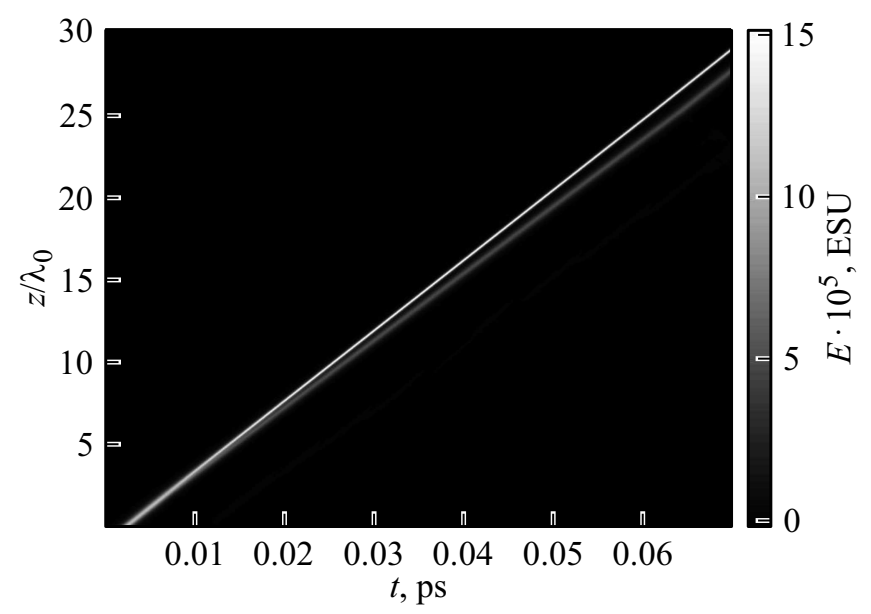

Рис. 1. Эволюция электрического поля субциклового импульса с исходной электрической площадью $4 \pi$ по мере распространения в двухуровневой резонансной среде. Видно разбиение исходного импульса на пару субцикловых импульсов.
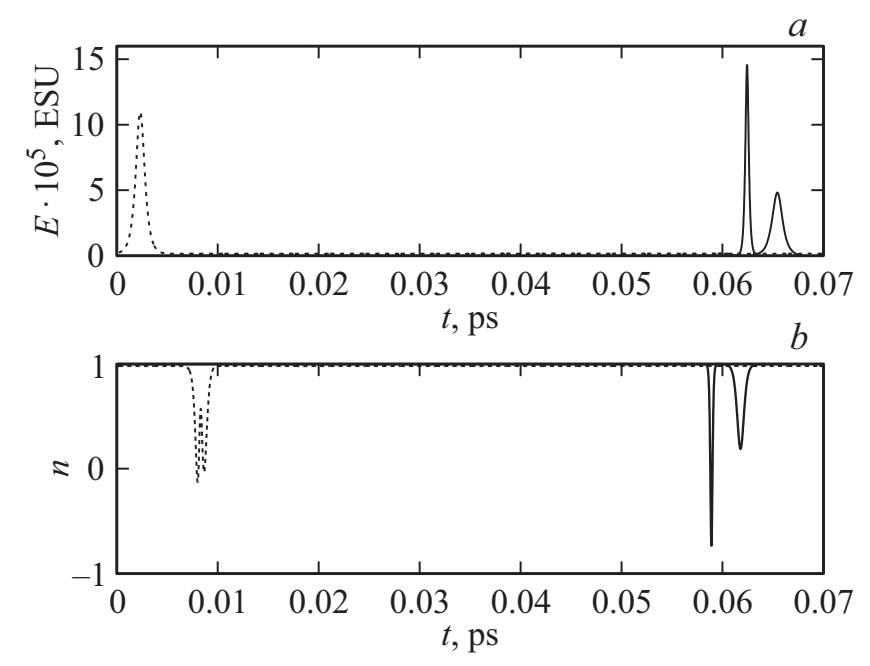

Рис. 2. (a) Временная зависимость электрического поля на входе в среду (пунктир) и на выходе (сплошная линия), (b) временная зависимость разности заселенностей на входе среды (пунктирная линия) и на выходе из среды (сплошная линия).

вой среде представлен на рис. 1. Параметры расчета приведены в таблице. Амплитуда импульса на входе выбиралась в виде $E_{0}=\frac{2 \hbar}{d_{12} \tau_{p}}$ чтобы его электрическая площадь равнялась $4 \pi$.

Из рис. 1 видно,что происходит расщепление входного импульса по мере распространения на два импульса разной амплитуды и длительности. Униполярность импульса практически сохраняется по мере распространения. Оба импульса движутся с разными скоростями в среде аналогично ситуации, когда длинный $4 \pi$-импульс расщепляется на пару $2 \pi$-импульсов СИП [16]. В результате на выходе из среды получается два субцикловых импульca, см. рис. 2, a, разной амплитуды и длительности.
Параметры, использованные при численном расчете

\begin{tabular}{l|c}
\hline Длина волны резонансного перехода & $\lambda_{0}=700 \mathrm{~nm}$ \\
\hline Дипольный момент перехода & $d_{12}=5 \mathrm{D}$ \\
\hline Время релаксации инверсии & $T_{1}=1 \mathrm{~ns}$ \\
\hline Время релаксации поляризации & $T_{2}=1 \mathrm{~ns}$ \\
\hline Концентрация атомов & $N_{0}=10^{21} \mathrm{~cm}^{-3}$ \\
\hline Амплитуда поля & $E_{0}=1.08 \cdot 10^{6} \mathrm{ESU}$ \\
\hline Длительность импульса & $\tau_{p}=388 \mathrm{as}$
\end{tabular}

Исходный импульс действует на среду на среду, как $4 \pi$-импульс, так как инверсия совершает две осцилляции, и среда возвращается в исходное состояние с инверсией 1 после прохождения импульса, см. рис. 2, a. Аналогично оба подимпульса, на которые расщепился исходный импульс, действуют на среду подобно $2 \pi$-импульсам, см. рис. $2, b$.

Аналогичное явление расщепления имеет место при распространении в среде униполярного импульса (6) с входной электрической площадью $6 \pi$.

\section{Заключение}

Таким образом, в настоящей работе изучено распространение субциклового униполярного импульса в форме гиперболического секанса с начальной электрической площадью $4 \pi$ (интеграл от электрического поля по времени (2)). В случае униполярных импульсов субцикловой длительности ввиду отсутствия у них медленной огибающей не применимо понятие площади импульса (1), пропорциональной интегралу от медленной огибающей импульса, которое используется при описании когерентного распространения длинных имульсов в резонансных средах.

Показано,что в случае распространения такого импульса в резонансной среде происходит расщепление исходного импульса на пару импульсов, каждый из которых действует на среду подобно $2 \pi$-импульсу. Это явление аналогично расщеплению длинных импульсов большой площади при когерентном распространении в резонансной среде.

Работа частично поддержана грантом РФФИ 16-02$00762 \mathrm{a}$.

\section{Список литературы}

[1] Krausz F., Ivanov M. // Rev. Mod. Phys. 2009. V. 81. P. 163.

[2] Manzoni C., Mücke O.D., Cirmi G., Fang S., Moses J., Huang S.-W., Hong K.-H., Cerullo G., Kärtner F.X. // Laser Photonics Rev. 2015. V. 9. P. 129.

[3] Calegari F., Sansone G., Stagira S., Vozzi C., Nisoli M. // J. Phys. B: At. Mol. Opt. Phys. 2016. V. 49. P. 062001. 
[4] Gallmann L., Cirelli C., Keller U. // Ann. Rev. Phys. Chem. 2012. V. 63. P. 447.

[5] Ramasesha K., Leone S.R., Neumark D.M. // Annu. Rev. Phys. Chem. 2016. V. 67. P. 41.

[6] Ciappina M.F., Pérez-Hernández J.A., Landsman A.S. // Rep. Prog. Phys. 2017. V. 80. P. 054401.

[7] Landsman A.S., Keller U. // Phys. Rep. 2015. V. 547. P. 1.

[8] Kozak M., McNeur J., Leedle K.J., Deng H., Schönenberger N., Ruehl A., Hartl I., Harris J.S., Byer R.L., Hommelhoff P. // Nat. Commun. 2017. V. 8. P. 14342.

[9] Skryabin D.V., Gorbach A.V. // Rev. Mod. Phys. 2010. V. 82. P. 1287.

[10] Dudley J.M., Genty G., Coen S. // Rev. Mod. Phys. 2006. V. 78. P. 1135.

[11] Leblond H., Mihalache D. // Phys. Rep. 2013. V. 523. P. 61.

[12] Mihalache D.U. // Roman. Rep. Phys. 2017. V. 69. P. 403.

[13] McCall S.L., Hahn E.L. // Phys. Rev. 1969. V. 183. P. 457.

[14] Крюков П.Г., Летохов В.С. // УФН. 1969. Т. 99. С. 169; Kryukov P.G., Letokhov V.S. // Sov. Phys. Usp. 1970. V. 12. P. 641 .

[15] Полуэктов И.А., Попов Ю.М., Ройтберг В.С. // УФН. 1974. T. 114. C. 97; Poluektov I.A., Popov Yu.M., Roitberg V.S. // Sov. Phys. Usp. 1975. V. 18. P. 673.

[16] Аллен Л., Эберли Дж. Оптический резонанс и двухуровневые атомы. М.: Мир, 1978; Allen L., Eberly J.H. Optical resonance and two-level atoms. NY: Wiley, 1975.

[17] Kalosha V.P., Herrmann J. // Phys. Rev. Lett. 1999. V. 83. P. 544.

[18] Xiao J., Wang Z., Xu Z. // Phys. Rev. A. 2002. V. 65. P. 031402

[19] Cai X., Zhao J., Wang Z., Lin Q. // J. Phys. B. 2013. V. 46. P. 175602.

[20] Lin Y., Chen I.H., Lee R.K. // Phys. Rev. A. 2011. V. 83. P. 043828 .

[21] Rosanov N.N., Semenov V.E., Vysotina N.V. // Laser Phys. 2007. V. 17. P. 1311.

[22] Высотина Н.В., Розанов Н.Н., Семенов В.Е. // Письма в ЖЭТФ. 2006. Т. 83. N 7. C. 337; Vysotina N.V., Rozanov N.N., Semenov V.E. // JETP Letters. 2006. V. 83. N 7. P. 279.

[23] Розанов Н.Н., Семенов В.Е., Высотина Н.В. // Квант. электрон. 2008. Т. 38. N 2. С. 137; Rosanov N.N., Semenov V.E., Vysotina N.V. // Quant. Electron. 2008. V. 38. N 2. P. 137.

[24] Высотина Н.В., Розанов Н.Н., Семенов В.Е. // Опт. и спектр. 2009. T. 106. N 5. C. 793; Vysotina N.V., Rosanov N.N., Semenov V.E. // Opt. Spectrosc. 2009. V. 106. N 5. P. 713.

[25] Kozlov V.V., Rosanov N.N., Angelis C.D., Wabnitz S. // Phys. Rev. A. 2011. V. 84. P. 023818.

[26] Розанов Н.Н. Диссипативные оптические солитоны. От микро- к нано- и атто-. М.: Физматлит, 2011. Гл. 17.

[27] Song X., Yang W., Zeng Z., Li R., Xu Z. // Phys. Rev. A. 2010. V. 82. N 5. P. 053821.

[28] Song X., Hao Z., Yan M., Wu M., Yang W. // Laser Phys. Lett. 2015. V. 12. P. 105003

[29] Архипов Р.М., Архипов М.В., Бабушкин И., Розанов Н.Н. // Опт. и спектр. 2016. Т. 121. N 5. C. 810; Arkhipov R.M., Arkhipov M.V., Babushkin I., Rosanov N.N. // Opt. Spectrosc. 2016. V. 121. N 5. P. 758.

[30] Arkhipov R.M., Arkhipov M.V., Babushkin I.V., Demircan A., Morgner U., Rosanov N.N. // Opt. Lett. 2016. V. 41. P. 4983.
[31] Архипов Р.М., Архипов М.В., Пахомов А.В., Бабушкин И., Розанов Н.Н. // Квант. Электрон. 2017. Т. 47. N 7. С. 589; Arkhipov R.M., Arkhipov M.V., Babushkin I., Pakhomov A.V., Rosanov N.N. // Quantum Electronics. 2017. V. 47. N 7. P. 589.

[32] Arkhipov R.M., Arkhipov M.V., Pakhomov A.V., Babushkin I., Rosanov N.N. // Las. Phys. Lett. 2017. V. 14. N 9. P. 1.

[33] Arkhipov R.M., Pakhomov A.V., Arkhipov M.V, Babushkin I., Demircan A., Morgner U., Rosanov N.N. // Sci. Rep. 2017. V. 7. N 1. P. 12467.

[34] Tarasishin A.V., Magnitskii S.A., Zheltikov A.M. // Opt. Commun. 2001. V. 193. P. 187.

[35] Tarasishin A.V., Magnitskii S.A., Shuvaev V.A., Zheltikov A.M. // Opt. Expr. 2001. V. 8. P. 452.

[36] Novitsky D.V. // Phys. Rev. A. 2011. V. 84. P. 013817.

[37] Novitsky D.V. // Phys. Rev. A. 2012. V. 85. P. 043813.

[38] Novitsky D.V. // J. Phys. B: At. Mol. Opt. Phys. 2014. V. 47. N 9. P. 095401.

[39] Novitsky D.V. // Opt. Commun. 2016. V. 358. P. 202.

[40] Архипов Р.М., Пахомов А.В., Архипов М.В., Бабушкин И., Толмачев Ю.А., Розанов Н.Н. // Письма в ЖЭТФ. 2017. T. 105. N 6. C. 388; Arkhipov R.M., Pakhomov A.V., Babushkin I., Tolmachev Yu.A., Rosanov N.N. // JETP Letters. 2017. V. 105. N 6. P. 408.

[41] Arkhipov R.M., Pakhomov A.V., Arkhipov M.V., Babushkin I., Tolmachev Yu.A., Rosanov N.N. // Laser Phys. 2017. V. 27. N 5. P. 053001.

[42] Розанов Н.Н. // Опт. спектр. 2009. T. 107. N 5. С. 761-765. Rosanov N.N. //Opt and spectr. 2009. V. 107. N 5. P.721.

[43] Bullough R.K., Ahmad F. // Phys. Rev. Lett. 1971. V. 27. C. 330 .

[44] Беленов Э.М., Назаркин А.В., Ущаповский В.А. // ЖЭТФ. 1991. T. 100. C. 762; Belenov E.M., Nazarkin A.V., Ushchapovskii V.A. // Sov. Phys. JETP. 1991. V. 73. P. 422.

[45] Архипов Р.М., Архипов М.В., Пахомов А.В., Бабушкин И., Розанов Н.Н. // Опт. и спектр. 2017. V. 123. N 4. С. 600; Arkhipov R.M., Arkhipov M.V, Pakhomov A.V., Babushkin I. // Opt. Spectrosc. 2017. V. 123. N 4. P. 610.

[46] Hughes S. // Phys. Rev. Lett. 1998. V. 81. N 16. P. 3363.

[47] Kozlov V.V., Rosanov N.N. // Phys. Rev. A. 2013. V. 87. N 4. P. 043836. 